\title{
Not miR-ly aging: SIRT1 boosts memory via a microRNA- dependent mechanism
}

\author{
Silvia Bicker ${ }^{1}$, Gerhard Schratt ${ }^{1}$ \\ ${ }^{I}$ Interdisziplinäres Zentrum für Neurowissenschaften, SFB488 Junior Group, Universität Heidelberg, and Institut für Neuroana- \\ tomie, Universitätsklinikum Heidelberg, Im Neuenheimer Feld 345, D-69120, Heidelberg, Germany \\ Cell Research (2010) 20:1175-1177. doi:10.1038/cr.2010.129; published online 7 September 2010
}

Long-term memory formation requires new synthesis of proteins that regulate synaptic plasticity. Recently, microRNAs (miRNAs) have been identified as master regulators of protein synthesis. miRNAs are an extensive class of small noncoding RNAs (21-25 nt) that preferentially bind to the $3^{\prime}$ untranslated regions (UTRs) of mRNAs, thereby either inducing translational repression or degradation of the target mRNA. In the nervous system, miRNAs control diverse processes, ranging from specification of neuronal cell identity to synaptic plasticity [1]. The potentially huge impact of miRNAs on memory formation is just beginning to be revealed. First evidence came from invertebrate systems, such as Aplysia and Drosophila [2, 3]. A recent study from the Tsai lab has now demonstrated that a miRNA-mediated mechanism plays a critical role in learning and memory in vertebrates [4]. Moreover, expression of this miRNA was discovered to be controlled by sirtuin 1 (SIRT1), a histone deacetylase well-known for regulating lifespan [5].

SIRT1 belongs to the sirtuin family, which has emerged as a key regulator of diverse processes closely related to aging, such as metabolism, gene expres-

Correspondence: Gerhard Schratt Tel: +49-6221-566210; Fax: +49-6221-567897 E-mail: schratt@ana.uni-heidelberg.de sion or genomic stability. Recent work linked SIRT1 to brain physiology and neurological disorders. In a previous study, Tsai and her team found that ectopic expression of SIRT1 improves neuronal survival in a mouse model of Alzheimer's disease (AD) [6]. The same neuroprotective effect was seen upon treatment with resveratrol, an activator of sirtuins. A recent study now showed that SIRT1 overexpression in AD mice suppresses $\beta$-amyloid production [7], one of the hallmarks of AD, emphasizing the beneficial activity of SIRT1 in neurodegeneration. However, the role of SIRT1 in memory storage remained unclear.

In their new study, Gao et al. investigated the role of SIRT1 in learning and memory [4]. Intriguingly, mice lacking SIRT1 displayed impaired cognitive abilities, such as associative memory, spatial learning or recognition memory. These deficits corresponded well to physical alterations in the brain. In the hippocampus of SIRT1 knock-out (KO) mice, the number of synapses was significantly decreased and longterm potentiation (LTP) was abrogated. These findings suggest that SIRT1 is an important regulator of synapse development and memory formation.

What could be the molecular mechanism used by SIRT1 to regulate memory? Since SIRT1 is a transcription factor, it is reasonable to look for changes in gene expression, with special focus on proteins involved in synaptic plasticity. Gao and co-workers analyzed the RNA and protein levels of cAMP responsive element binding protein (CREB), a key regulator of synapse formation. Interestingly, the protein levels of CREB were significantly downregulated in SIRT1 KO mice, whereas CREB mRNA levels were not decreased. These data indicate a post-transcriptional regulation of CREB gene expression. Since miRNAs are emerging as key modulators of post-transcriptional gene regulation, the researchers looked for changes in miRNA levels. In fact, the expression of several brain-enriched miRNAs was altered in SIRT1 deficient mice. Among them, miR-134 was of special interest, since previous work showed that miR134 controls spine morphogenesis and dendritogenesis in hippocampal neurons by regulating local protein synthesis at synaptic sites [8]. Spine size is a good correlate of synaptic strength and therefore directly linked to memory storage.

Next, Tsai and her team found that SIRT1 is part of an inhibitor complex that negatively regulates miR-134 expression by binding to two regulatory elements upstream of the miR-134 gene. As a consequence, SIRT1 KO mice have aberrantly high miR-134 levels, causing enhanced translational repression of its target mRNAs. Sequence analysis 


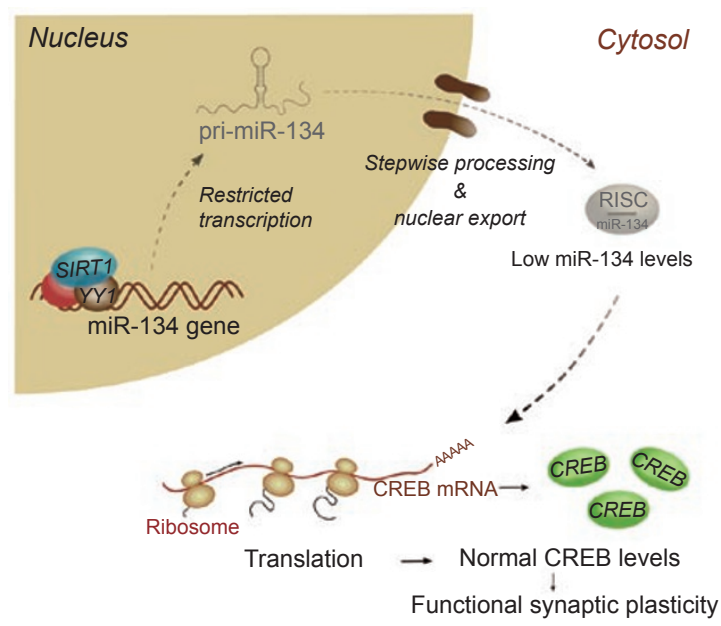

A Normal physiological conditions

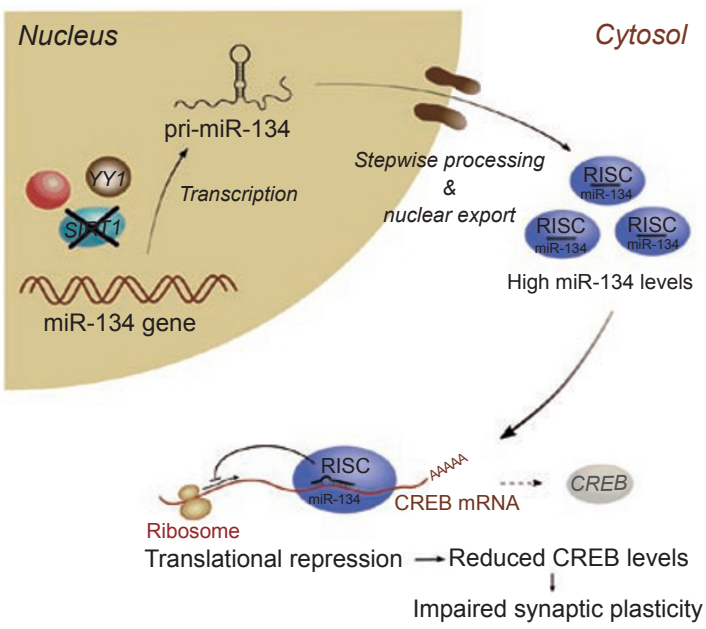

B SIRT1 loss of function

Figure 1 Model for the regulation of synaptic plasticity by SIRT1. (A) Under normal physiological conditions, SIRT1 cooperates with $\mathrm{YY} 1$ and possibly other proteins, leading to restricted expression of miR-134. Concomitant high levels of CREB enable functional synaptic plasticity. (B) SIRT1 loss-of-function causes aberrantly high expression levels of miR-134. Binding of miR-134 to complementary sites in the 3'UTR of CREB mRNA blocks synthesis of CREB protein and causes impaired synaptic plasticity.

revealed three miR-134 binding sites in the 3'UTR of CREB mRNA. In line with the known inhibitory effect of miRNAs on protein synthesis, overexpression of miR-134 in wild-type mice led to reduced CREB levels, accompanied by abrogated LTP and impaired memory. On the other hand, blocking miR-134 in SIRT1 KO mice could restore LTP and memory formation.

In conclusion, an inhibitor complex consisting of SIRT1, the sequencespecific transcription factor Yin Yang 1 (YY1) and possibly additional proteins restricts the expression of miR-134 under normal physiological conditions. Consequently, SIRT1 loss-of-function causes higher levels of miR-134 and a concomitant translational block of miR-134 targets such as CREB, thereby interfering with synaptic plasticity (Figure 1). This study by Gao and colleagues is the first to reveal that besides its previously known anti-aging activity, SIRT1 also plays a role in synaptic plasticity, learning and memory. Meanwhile, a similar study by Michán et al. proved that SIRT1 deficiency causes cognitive impairment in mice [9]. Based on these data, it is tempting to speculate that SIRT1 represents a promising target for the treatment of neurodegenerative diseases. Furthermore, to deepen our understanding of the underlying cellular mechanisms, it will be interesting to disentangle the signaling pathways that regulate the function of the SIRT1 containing inhibitor complex under normal physiological conditions.

Finally, this work clearly reinforces the importance of miRNAmediated mechanisms in the brain. The miR-134 gene is part of a large cluster of brain-specific miRNAs (miR379-410), which is expressed as a polycistronic transcript [10]. Our lab previously demonstrated that transcription of this cluster is induced by neuronal activity and mediated via binding of Mef2 to a regulatory element located $\sim 15 \mathrm{~kb}$ upstream of miR379-410 [11]. In comparison, the YY1 DNA binding sites were found to be $\sim 5 \mathrm{~kb}$ upstream of the miR-134 gene. Further experiments are needed to clarify the influence of SIRT1 on the expression of other miRNAs from this cluster. In summary, this study provides the first link between SIRT1 and miRNAs in memory formation. An intriguing question is the potential role of miRNA-based mechanisms in other processes regulated by SIRT1, such as neurodegeneration or aging. Since perturbations in miRNA expression can manifest in a wide range of CNS dysfunctions, miRNA-based therapeutics have great potential for the treatment of neurological disorders. Undoubtedly, future studies within the field of brain miRNAs will expand our understanding of the complex mechanisms underlying higher cognitive functions and neurological diseases.

\section{References}

1 Kosik KS. The neuronal microRNA system. Nat Rev Neurosci 2006; 7:911920.

2 Rajasethupathy P, Fiumara F, Sheridan $\mathrm{R}$, et al. Characterization of small RNAs in aplysia reveals a role for miR124 in constraining synaptic plasticity through CREB. Neuron 2009; 63:803817. 
3 Ashraf SI, McLoon AL, Sclarsic SM, Kunes S. Synaptic protein synthesis associated with memory is regulated by the RISC pathway in Drosophila. Cell 2006; 124:191-205.

4 Gao J, Wang WY, Mao YW, et al. A novel pathway regulates memory and plasticity via SIRT1 and miR134. Nature 2010 Jul 11. doi:10.1038/ nature 09271

5 Finkel T, Deng CX, Mostoslavsky R. Recent progress in the biology and physiology of sirtuins. Nature 2009; 460:587-591.
6 Kim D, Nguyen MD, Dobbin MM, et al. SIRT1 deacetylase protects against neurodegeneration in models for Alzheimer's disease and amyotrophic lateral sclerosis. EMBO J 2007; 26:31693179.

7 Donmez G, Wang D, Cohen DE, Guarente L. SIRT1 suppresses betaamyloid production by activating the alpha-secretase gene ADAM10. Cell 2010; 142:320-332.

8 Schratt G. microRNAs at the synapse. Nat Rev Neurosci 2009; 10:842-849.

9 Michan S, Li Y, Chou MM, et al. SIRT1 is essential for normal cognitive function and synaptic plasticity. $J$ Neurosci 2010; 30:9695-9707.

10 Seitz H, Royo H, Bortolin ML, Lin SP, Ferguson-Smith AC, Cavaillé J. A large imprinted microRNA gene cluster at the mouse Dlk1-Gtl2 domain. Genome Res 2004; 14:1741-1748.

11 Fiore R, Khudayberdiev S, Christensen $\mathrm{M}$, et al. Mef2-mediated transcription of the miR379-410 cluster regulates activity-dependent dendritogenesis by fine-tuning Pumilio2 protein levels. EMBO J 2009; 28:697-710. 